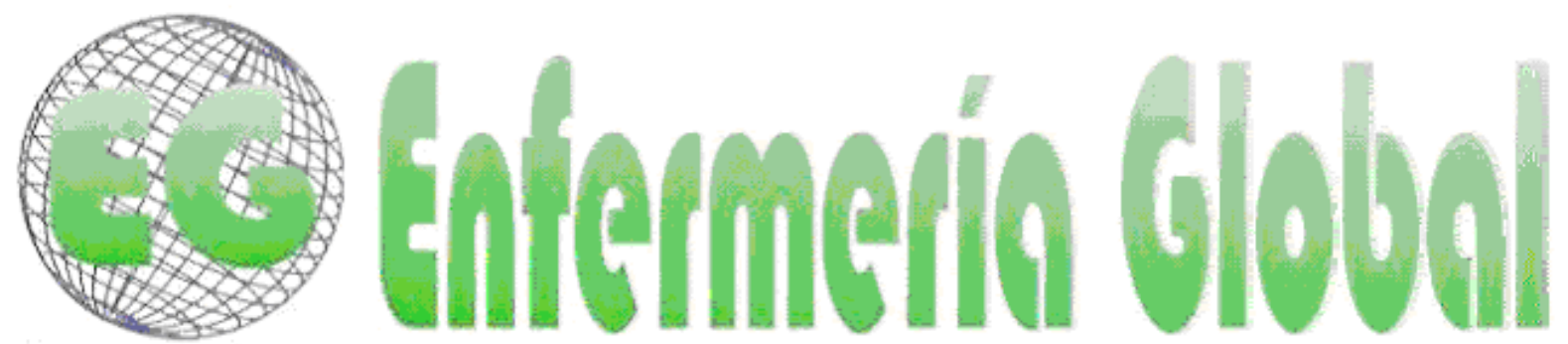

\title{
Las Fan Page de Facebook como medio de difusión de la Enfermería Basada en la Evidencia
}

Facebook pages for the broadcasting of media on evidenced-based nursing

\author{
*Santillán García, A. **Cornejo Marroquín, C. **Ausín Lomas, S. \\ *Enfermera asistencial y docente. Autora del blog "Enfermeria Basada en la Evidencia" y responsable \\ de su difusión por las redes sociales. E-mail: ebevidencia@gmail.com **Supervisora de Área. \\ Complejo Asistencial Universitario de Burgos (CAUB) \\ Palabras clave: blog; internet; innovación; eSalud; social media
}

Keywords: stress; blog; internet; innovation; eHealth; social networking.

\section{RESUMEN}

Desde el cambio que Internet sufrió a principios de este siglo evolucionando hacia la Web 2.0 se ha generado un cambio de paradigma en la manera de gestionar la información. Este hecho no le ha sido indiferente a la enfermería, que está aprendiendo a utilizar las herramientas 2.0 entre otras motivaciones, en beneficio de su difusión científica. A su vez, las redes sociales han cobrado un protagonismo tal que la combinación de redes sociales y herramientas 2.0 forman el escenario actual de la web social. Facebook y sus FanPages forman parte de esta web social y pueden estar al servicio de la ciencia fomentando la difusión de la información y la comunicación. En este estudio descriptivo se muestra la difusión de los contenidos del blog "Enfermería Basada en la Evidencia" a través de la FanPage de su perfil público en Facebook, que ha tenido un alcance rápido y transcultural. Esto nos describe el marco social de la web 2.0 al servicio de la ciencia.

\begin{abstract}
Since the change that the Internet suffered earlier this century evolving into Web 2.0, a change in the model of how to manage information has been generated. This fact has not been indifferent to nurses, who are learning how to use 2.0 tools, among other motivations, for the benefit of scientific broadcasting. In turn, social networks have taken on a role such that the combination of social networks and 2.0 tools are the current scenario of the social web. Facebook and its Fan Pages are part of the social web and can be used by science for promoting the communication of information. This descriptive study shows the spreading of the contents of the blog called "Evidence-Based Nursing" through its Facebook page, which has a rapid and cross-cultural range. This describes the social context of Web 2.0 in the service of science.
\end{abstract}




\section{INTRODUCCIÓN}

En los últimos tiempos, conceptos como Web 2.0, blog y redes sociales aparecen con frecuencia en el ámbito de la salud y de la enfermería ${ }^{1}$. Facebook es una de estas redes sociales y sus FanPage (o páginas) facilitan la difusión de contenidos. Si estos contenidos están directamente relacionados con la Enfermería Basada en la Evidencia, las FanPage se pueden convertir en difusoras del conocimiento enfermero.

\section{MARCO TEÓRICO}

A principios del siglo XXI, Internet sufrió un cambio muy significativo evolucionando hacia la Web $2.0^{2}$ y así se generó un cambio de paradigma en la manera de gestionar la información

En los inicios de Internet, el usuario estaba limitado a la consulta u observación de contenidos, con unas posibilidades reducidas de interacción tanto con la web como con otros usuarios. Con la Web 2.0, Internet se convierte en una plataforma de inteligencia colectiva en donde se genera, comparte y debate información. Este proceso se ve favorecido por la utilización del software ligero y sin limitaciones que rompe el ciclo de las actualizaciones.

Este hecho ha tenido repercusiones en el ámbito de la salud, apareciendo el concepto de Salud $2.0^{3}$, eSalud o eHealth. Con la aparición de nuevas herramientas como los webinars, las wikis, las redes sociales, chats, etc,. los usuarios (como pueden ser los profesionales de salud, pacientes y familiares) pueden crear información, compartirla, evaluarla y difundirla pasando de ser un consumidor pasivo de información a un prosumidor (prosumer, en inglés) neologismo que suma la acción del productor y consumidor.

Si la Web 2.0 hace referencia a un grupo de tecnologías que facilitan la conexión social, y donde "todos y cada uno -de los usuarios se entiende- son capaces de añadir y editar la información" ${ }^{4}$, nos faltaría precisar a qué tecnologías o herramientas nos referimos. El propio O'Reilly ${ }^{2}$ las define como Software Social y entre sus características más importantes está la de convertir al usuario en contribuyente informador, el afianzamiento de la noción del poder de las multitudes y el enriquecimiento en la experiencia del usuario.

Como ejemplos paradigmáticos de este tipo de herramienta 2.0 tenemos los Blogs (de los que se va a hablar de manera más extensa en párrafos posteriores) y las Wikis, pero existen otras muchas tecnologías que ofrecen más posibilidades y de hecho aparecen herramientas nuevas casi a diario.

A estas herramientas se las puede clasificar ${ }^{5}$ según su funcionalidad y a efectos prácticos podemos hablar de herramientas de publicación (por ejemplo Blogger o Slideshare), herramientas de etiquetados o marcado social (como Delicious) y herramientas de edición y construcción colaborativa de recursos (por ejemplo, GoogleDocs).

Estas herramientas 2.0 o también llamadas herramientas sociales, poco a poco inundan nuestro entorno informático, conectándose unas con otras y favoreciendo el 
intercambio de contenidos entre los usuarios. A este flujo de información es a lo que se denomina inteligencia colectiva, y nace de la web social ${ }^{6}$.

La relación de la web social con la ciencia ha sido explicada por autores como explica Codina ${ }^{7}$. Según éste, la ciencia es comunicación y la ciencia es colaboración. Sin ambos ingredientes parece imposible el avance de las investigaciones, ni su divulgación. No tiene sentido que se realicen estudios y se trabaje en investigaciones si los resultados no son divulgados y utilizados por la comunidad científica. Esta idea fue magníficamente expuesta por los fundadores de Research Gate ${ }^{8}$ en su plataforma de colaboración dirigida a científicos de todas las disciplinas. La plataforma ofrece acceso gratuito a las aplicaciones Web 2.0 más modernas. También evidencia cómo ambas cosas (comunicación y colaboración) pueden mejorar con el uso de instrumentos como las redes sociales. Estas dos ideas están muy bien expresadas por los fundadores de ResearchGate:

"The vision of Science 2.0 is promising: Communication between scientists will accelerate the distribution of new knowledge. [...] Science is collaboration, so scientific social networks will facilitate and improve the way scientists collaborate. Cooperation on scientific publications can be facilitated through Wiki-like concepts (ResearchGate, 2009)".

De esta contribución se deriva que el binomio comunicación y colaboración confluyen en el concepto de ciencia 2.0 y que esta ayuda a los científicos a difundir su conocimiento.

Como parte activa de esta Web Social, está la figura de las redes sociales digitales, o comunidades virtuales. Cierto es que las comunidades de práctica se han descrito como niveles superiores de relaciones humanas en comparación con las redes sociales porque implican un nivel mayor de compromiso entre los componentes, un objetivo común y un código común que favorezca las interrelacciones ${ }^{9}$. Pero aún existen divergencias entre los propios componentes de estos grupos y persisten las discrepancias en cuanto a la auto denominación de estos grupos virtuales. ${ }^{10}$

Las interacciones sociales mediadas por las Tecnologías de la Información y la Comunicación en el contexto de la sociedad de la información han sido estudiadas ${ }^{14}$ desde perspectivas sociológicas y antropológicas. A este respecto los expertos opinan que no se debe desvincular el plano de la denominada "realidad física" del plano de la realidad virtual, dado que ambas tienen componentes convergentes (y divergentes). De hecho, el interés por estas interacciones sociales en forma de comunidades de práctica 2.0 en el entorno de los profesionales de la salud está aumentando dado que son un interesante método de aprendizaje y formación continuada y elementos que favorecen la colaboración y el trabajo en equipo ${ }^{11}$.

Por otro, las redes sociales digitales populares como Facebook y Twitter cada vez están más presentes en nuestras vidas. Sus utilidades son variadas pero sin duda se muestran día a día como potentes herramientas de comunicación.

Es un hecho que el uso de las redes sociales se está extendiendo significativamente en el ámbito de la salud. Tanto los profesionales como las instituciones, organizaciones, asociaciones y pacientes utilizan las redes para difundir su labor, compartir conocimiento y generar inteligencia colectiva ${ }^{12}$. 
Según los últimos informes publicados en España ${ }^{13,14}$ las principales redes sociales en nuestro ámbito geográfico son en primer lugar Facebook (utilizado por 8 de cada 10 internautas), seguido de Twitter (que reduce su media de edad y crece en usuarios gracias a la incorporación de un público más joven y se convierte en la red con más expectativas de crecimiento) y Tuenti (que se consolida como la segunda gran red social de España).

Respecto a nuevos medios de comunicación cabe mencionar la figura del blog. Un blog (también llamado Weblog) es una bitácora en donde uno o varios autores escriben textos llamados post y quedan publicados en la red. Las experiencias de las enfermeras con el uso de los blogs como herramientas de mejora de la comunicación son positivas ${ }^{16}$ y se ha visto que más de la mitad de los blogs sanitarios estadounidenses están escritos por personal sanitario ${ }^{17}$.

La utilidad de los blogs tiene especial interés en al campo de la formación de enfermería dado que son herramientas de fácil utilización. Su uso favorece el proceso de aprendizaje de los estudiantes de enfermería ya que les ayuda a desarrollar sus habilidades en la esfera de la comunicación ${ }^{18}$.

El interés de los sanitarios en los usos y características de los usos y características de los blogs ${ }^{19}$ origina que la blogosfera sanitaria en español sea heterogénea. Esta engloba a profesionales sanitarios de categorías diversas, y al igual que ocurre en otras redes sociales, tiene representación de instituciones y organismos oficiales ${ }^{20}$.

Dado que existen antecedentes en España que señalan que un blog sanitario es un poderoso y barato medio de comunicación ${ }^{21}$ y por la fenomenología presentada, se creó un blog con el objetivo de difundir la metodología de la Enfermería Basada en la Evidencia y de fomentar las prácticas basadas en evidencias. A su vez, se crearon perfiles para el blog en Facebook, Twitter y Tuenti así como una Fan Page en Facebook. Se vincularon los contenidos del blog a las redes sociales mediante herramientas específicas (widgets).

El 21 de Julio de 2010 comenzó la andadura del perfil público en Facebook del blog "Enfermería Basada en la Evidencia" (www.ebevidencia.blogspot.com). Simultáneamente se creó la (https://www.facebook.com/home.php\#!/pages/Enfermeria-Basada-en-la-

Evidencia/104505709603682) para dar cobertura a las personas que quisieran seguir los contenidos publicados sin compartir sus perfiles. En este tiempo, el perfil ha generado más de 4000 "amigos" y la Fan Page 750 "fans", y 350 seguidores vía "me gusta". En total, son más de 5000 personas las que han elegido Facebook como vía de actualización de sus conocimientos en cuanto a la metodología de la enfermería basada en evidencias. Varios estudios muestran cómo las redes sociales son un medio utilizado por profesionales y pacientes para buscar información sobre salud. Los científicos debemos tener muy en cuenta el papel de estos nuevos medios en la trasmisión de información científica. Mediante la automatización de contenidos con las aplicaciones y las conexiones directas entre las páginas web y las redes sociales, se consigue una mayor difusión y que esta sea personalizada.

\section{OBJETIVO}

Describir el alcance y la difusión de los contenidos publicados en la Fan Page de "Enfermería Basada en la Evidencia". 


\section{EBevidencia:}

Enfermeria Basada en la Evidencia

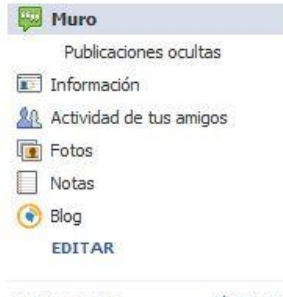

Información Editar

FanPage del sitio "Enfermeria

Basada en la Evidencia"

ver más

A

1.035

personas les gusta esto

19

personas están hablando de esto

Me gusta Ver todos

C. Observatorio

para la Seguridad

del Paciente

Biblioteca Virtual del Sistema
Sanitario Público

Medicina Basada Medicina Basada Tecnológico de

Biblioteca Médica

ciencia - Editar información

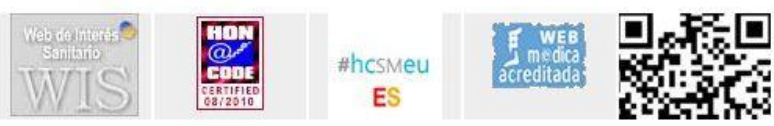

Muro

Enfermeria Basada en la E.... · Todos (Más recientes)

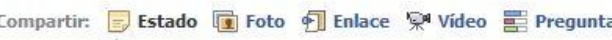

Escribe algo...

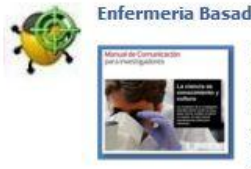

Manual de comunicacion para investigadores

Cuando te decides a divulgar lo que bien sabes, en lo que te

has especializado o lo que has investigado, muchas veces te

encuentras con miles de barreras que no sabes por dónde

sortear. Si esto te ha pasado o te sucede ahora, toma nota

de lo siguiente, La Universidad de la Rioja $p$

link: Full Artide...

589 impresiones $\cdot 1,02 \%$ de comentarios

- Me gusta - Comentar - Share - El Lunes a las 0:00 a través de NetworkedBlogs

[3 A 7 personas les gusta esto.

Escribe un comentario

Enfermeria Bas

oticias del corazón (rehabilitado)

Enfermeria en Cardiologia (al cual tengo el placer de

ovedades interesantes. En primer lugar, el artículo de la

Sulce: Enfermeria Basada en la Evidencia

\section{MATERIAL Y MÉTODOS}

Estudio descriptivo, observacional y retrospectivo sobre los resultados analíticos de la Fan Page del blog "Enfermería Basada en la Evidencia" en Facebook.

El ámbito de estudio ha sido la Fan Page mencionada y la población fueron todas aquellas personas que en el momento de la medición de los datos se habían suscrito a los contenidos de dicha Fan Page a través del botón "me gusta" (N=738).

Los datos se recogieron directamente de la página de estadísticas de Facebook (Facebook Insights). Se realizó análisis univariante, con presentación de estadísticos descriptivos (porcentajes).

Los contenidos de la Fan Page corresponden a las entradas del blog mencionado, actualizados automáticamente mediante la aplicación gratuita "NetworkedBlogs". Los datos descritos se han recogido entre el 1 de Agosto de 2010 y el 1 de Junio de 2011. Las variables estudiadas se han agrupado en 3 bloques: usuarios, contenidos e interacciones.

\section{RESULTADOS}

Usuarios: 738 perfiles se han agregado a la Fan Page. La media de usuarios activos por meses de 317 (entendiéndose por usuario activo aquel que abre al menos un enlace de la página). El $75 \%$ corresponde a perfiles femeninos y el $24 \%$ masculino, siendo más frecuente el rango de edades de los 25 a 34 años en mujeres y de 35 a 
44 en hombres. La ubicación geográfica de los seguidores es mayoritariamente España $(n=308)$ seguido de Argentina $(n=166)$ y Venezuela $(n=124)$.

Contenidos: Los contenidos publicados son los post del blog "Enfermería Basada en la Evidencia". Corresponden al $100 \%$ con los 99 post publicados en el blog. El método de publicación de los contenidos estaba automatizado desde la propia página del blog y su conexión con Facebook. Ningún post se ha perdido, duplicado o modificado debido a la automatización.

Interacciones: 44221 visualizaciones y 140 comentarios. El contenido más visualizado corresponde al título "Estrategias de implementación" y el que más feed back ha tenido ha sido "Cuidados de salud participativos".

\section{DISCUSIÓN}

La mayoría de los usuarios (75\%) son mujeres y esta cifra parece estar en consonancia con la registrada en España por el INE en $2010\left(83^{\prime} 3 \%\right)^{22}$. Además la franja de edad de los fans de la página coincide con las ultimas tendencias de uso de internet y las TIC en España ${ }^{23}$.

Consideramos que el total de usuarios que interactúan con la Fan Page es uno de los datos más reveladores acerca del funcionamiento de la página, dado que los usuarios pasivos (aquellos que clican una vez en "me gusta" y no vuelven a interactuar) no podemos asegurar que accedan a los contenidos. En el caso de estudio, la media de usuarios activos al mes se aproxima al $50 \%$.

El método de publicación automatizado de los contenidos ha resultado eficaz al no perder, duplicar ni modificar ningún post. Esto unido su fácil e intuitivo manejo nos anima a recomendar su utilización.

El número de visualizaciones de los contenidos es elevado. Las visualizaciones directas de los contenidos a través del propio blog en periodo similar fue de más de $43000^{24}$, así que la Fan Page duplica la visibilidad de los contenidos del blog "Enfermería Basada en la Evidencia". No coinciden sin embargo los contenidos más visitados por ambos medios.

\section{Limitaciones}

Facebook Insights no está disponible para todas las cuentas, siendo requisito a día de hoy que una Fan Page cuente con al menos 30 fans para poder utilizar su estadística. La explotación de datos a través de Facebook todavía está muy restringida pero el equipo técnico de Facebook asegura su significancia estadística.

\section{CONCLUSIONES}

Los contenidos publicados en Facebook llegan rápidamente a las personas interesadas en esa información. El alcance es transcultural y se puede gestionar mediante herramientas gratuitas.

Dado que los pacientes cada vez utilizan más este medio para buscar información y soporte en cuanto a salud, y que los propios profesionales también recurren a esta red para mantenerse actualizados; Facebook y sus recursos son elementos a tener 
en cuenta por las enfermeras tanto para la comunicación entre pares como para la educación para la salud a los ciudadanos.

Llegados a este punto, cabe reflexionar sobre la necesidad de regulación del uso de las redes sociales por parte de los profesionales sanitarios ${ }^{25}$ y de los estudiantes ${ }^{26}$. No cabe duda de que la libertad de expresión de los individuos es incuestionable, pero es necesario tener en cuenta que cuando se utilizan entornos institucionales, cuando se interactúa como profesional sanitario es necesario tener presentes ciertas normas de usos y estilo como las ya divulgadas por el País Vasco ${ }^{27}$, la Generalitat de Cataluña ${ }^{28}$ o el Hospital materno infantil Hospital Sant Joan de Déu ${ }^{29}$.

\section{REFERENCIAS BIBLIOGRÁFICAS}

1- Lau, AS. Hospital-based nurses' perceptions of the adoption of web 2.0 tools for knowledge sharing, learning, social interaction and the production of collective intelligence. J Med Internet Res. 2011 Nov 11; 13(4):e92. [Consultado 08 Dic. 2011]. Disponible en: http://www.jmir.org/2011/4/e92/

2- O'Reilly [página principal en internet]. What is Web 2.0. Design patterns and business models for the next generation of software. [actualizado 30 Sep. 2005; citado 02 sep. 2011]. Disponible en: http://oreilly.com/web2/archive/what-isweb-20.html

3- Lorca J, Jadad A. ¿Salud 2.0? Revista e Salud. 2009; 5(19).

4- Anderson $P$. What is Web 2.0? Ideas, technologies and implications for education. JISC Technology and Standards Watch, Feb. 2007. [Consultado 08 Dic. 2011]. Disponible en: www.jisc.ac.uk

5- Castañeda Quintero, L. Software social para la escuela 2.0: más allá de los Blogs y las Wikis. En: Inclusión Digital en la Educación Superior: Desafíos y oportunidades en la sociedad de la Información. X Congreso Internacional EDUTEC. [Consultado 08 Sep. 2011]. Disponible en: http://lindacastaneda.com

6- Ribes, X. La Web 2.0. El valor de los metadatos y de la inteligencia colectiva. Rev.Telos. Oct-Nov 2007. № 73. [Consultado 10 Dic. 2011].

7- Codina L. Ciencia 2.0: Redes sociales y aplicaciones en línea para académicos [on line]. "Hipertext.net", núm. 7, 2009. http://www.hipertext.net [Consulta: 08 Sep. 2011]. ISSN 1695-5498

8- ResearchGate [página principal en internet]. [Consultado 02 Sep. 2011]. Disponible en: http://www.researchgate.net/

9- Wenger E. Communities of Practice: Learning, Meaning, and Identity. New York: Cambridge University Press; 1998.

10-Santillán A, Zurbarán M, Sánchez F. Enfermería Basada en la Evidencia Blog. Resultado de la encuesta "Definiendo al grupo de salud 2.0" [Internet]. Burgos. [Consultado 22 Sep. 2011]. Disponible en: http://ebevidencia.blogspot.com/2011/09/resultado-de-la-encuesta-definiendoal.html

11-Sandoval, E A. Cibersocioantropología de comunidades virtuales. Rev. argent. sociol. [online]. 2007, vol.5, n.9 [citado 2011-09-22], pp. 64-89. Disponible en: http://redalyc.uaemex.mx

12-Navarro Martínez O. Comunidades de práctica 2.0 en el entorno de profesionales de la salud. Enferm Clin. Vol 21, Issue 5, September-October 2011, Pages 235-237 
13-Fernández L, Traver V. La nueva sanidad y los nuevos pacientes en la Web 2.0 [monografía en Internet]. Diario Médico; 2010 [Consultado 03 Sep. 2011].

Disponible en: http://www.diariomedico.com/

14--Informe de resultados del Observatorio de Redes Sociales en España: $2^{\mathrm{a}}$ oleada (Febrero 2011) [Consultado 03 Sep. 2011]. Disponible en: http://www.tcanalysis.com/.

15-The Cocktail analysis. Tercera oleada del observatorio de redes sociales. [Consultado 04 Mar 2011] Disponible en: http://www.tcanalysis.com/.

16-Farr T. Using a blog to improve communication. Nurs Manage. 2011 Jun; 42(6):52-3.

17-Miller EA, Pole. A. Diagnosis blog: checking up on health blogs in the blogosphere. Am J Public Health. 2010 Aug; 100(8):1514-9.

18-Maag, M.et al. The potential use of blogs in nursing education. Comput Inform Nurs. 2005 Jan-Feb; 23(1):16-24; quiz 25-6.

19-Guimarães F. Blog - o que é? Como funciona? E por que "blogar"? Radiol Bras. $2011 \mathrm{Mai} / J u n ; 44(3): \mathrm{VII}-\mathrm{VIII}$

20-Weblogs Sanitarios en Español. [Consultado 22 de Sep. de 2011] Disponible en: http://www.infodoctor.org/rss/rss/

21-C. Álvarez Rodríguez, C. et al. Valoración de la eficacia de un blog de medicina de urgencias como medio de comunicación. Emergencias 2007; 19:180-186. [acceso de 28 de Jul. de 2011] Disponible en http://www.semes.org/revista/vol19 4/3.pdf

22-España en cifras 2010. Instituto Nacional de Estadística. [Consultado 10 de Dic. de 2011] Disponible en: www.ine.es/prodyser/pubweb/espcif/espcif10.pdf

23-Encuesta sobre equipamiento y uso de tecnologías de información y comunicación en los hogares. 2010. Instituto Nacional de Estadística.

[Consultado 10 de Dic. de 2011] Disponible en: www.ine.es

24-Santillán A. Valoración de un blog como elemento de comunicación de enfermería. XV Encuentro Internacional de Investigación en Cuidados. Poster. Libro de ponencias. Pag 467-468. [Consultado 15 de Dic. de 2011] Disponible en: http://www.isciii.es/

25-Livingstone P, Vogel E.I. Facebook and the Registered Nurse. SRNA NewsBulletin. SUMMER 2010.

26-Skiba, Diane I. Nursing Education 2.0: the need for social media policies for schools of nursing. Nursing Education Perspectives. Mar 2011. ISSN: 15365026.

27-Guía de usos y estilo en las Redes Sociales del Gobierno Vasco. [en línea].Vitoria-Gasteiz: Servicio Central de Publicaciones del Gobierno Vasco. 2011. Disponible en: www.irekia.euskadi.net

28-Guía de usos y estilo en las redes sociales de la Generalidad de Cataluña [en línea]. Barcelona: Generalidad de Cataluña. Departamento de la Presidencia, 2010. Disponible en: www.gencat.cat. [Consultado 19 de Sep. de 2011].

29-Política de redes sociales del Hospital Sant Joan de Déu - Barcelona [en línea]. Barcelona: Hospital materno infantil Sant Joan de Déu. Universitat de Barcelona, 2011. Disponible en: www.hsjdben.org [Consulta: 19 de Sep. de 2011].

ISSN 1695-6141

(C) COPYRIGHT Servicio de Publicaciones - Universidad de Murcia 